\title{
PERIODONTITE E A INFECCCÃO PELO SARS-COV-2 EM PACIENTES COM DIABETES MELLITUS: UMA REVISÃO DA LITERATURA
}

\section{Marina Monteiro Rossato'; Lilian Oliveira de Oliveira ${ }^{2}$; Elisangela Colpo ${ }^{3}$; Minéia Weber Blattes ${ }^{4}$}

\section{RESUMO}

O objetivo deste trabalho foi realizar uma revisão da literatura com o propósito de encontrar materiais relevantes e atualizados sobre a relação da periodontite em pacientes com diabetes mellitus (DM) infectados pela COVID-19. O DM é uma doença crônica determinada pela perda do controle da homeostase da glicose, que pode afetar os órgãos do corpo. Observou-se que o DM é uma condição que aumenta a gravidade das doenças bucais, e a doença está associada de maneira bilaterial com a periodontite, isto é, a periodontite pode ser uma complicação do DM por descontrolar os níveis glicêmicos e ter DM aumenta a possibilidade de desenvolver periodontite. Os pacientes com DM infectados com o SARS-CoV-2, apresentam maiores chances de desenvolver complicações, inclusive a periodontite, ressaltando a importância da higiene bucal nesses pacientes.

Palavras-chave: Coronavírus, COVID-19, Odontologia.

Eixo Temático: Atenção Integral e Promoção à Saúde

\section{INTRODUÇÃO}

O diabetes mellitus (DM) é uma doença endócrino-metabólica de etiologia heterogênea, que envolve fatores genéticos, biológicos e ambientais, caracterizada por hiperglicemia crônica na ausência de tratamento. Resultante de defeitos parciais ou totais na secreção ou na ação da insulina, onde o indivíduo pode apresentar riscos e complicações sistêmicas, dentre elas complicações orais (SBD, 2019).

O DM é um fator de risco para doenças gengivais e está associado de forma bilateral. Pacientes com DM aumentam a possibilidade de desenvolver periodontites,

\footnotetext{
${ }_{1}^{1}$ Acadêmica do Curso de Odontologia. Bolsista PROBEX - Universidade Franciscana/UFN marina.rossato@ufn.edu.br

2 Professora do Curso de Fisioterapia - Universidade Franciscana/UFN - lilian.oliveira@ufn.edu.br

3 Professora do Curso de Nutrição - Universidade Franciscana/UFN - elicolpo@ufn.edu.br

4 Orientadora. Professora do Curso de Biomedicina - Universidade Franciscana/UFN -

mweber@ufn.edu.br
} 
o que pode acarretar uma complicação do DM nos pacientes com a doença que apresentam variabilidade glicêmica, pois induzem a inflamação sistêmica, que por sua vez aumenta ou reforça a resistência crônica à insulina (BRANDÃO; SILVA; PENTEADO, 2011).

A pandemia causada pelo vírus SARS-CoV-2 impactou toda a população mundial e estudos mostram que o DM é um preditor significativo para desenvolvimento da COVID-19. A associação entre DM e aumento da mortalidade por COVID-19 podem estar relacionadas aos efeitos sistêmicos adicionais da periodontite (PFÜTZNER; LAZZARA; JANTZ, 2020).

O DM é um importante problema de saúde em toda a população mundial, e está aumentando constantemente, desta forma, o objetivo deste trabalho de revisão da literatura foi analisar a relação da periodontite em pacientes infectados pelo COVID-19 e com diagnóstico de DM.

\section{METODOLOGIA}

Foi realizada uma revisão da literatura com o propósito de encontrar materiais relevantes e atualizados sobre as complicações odontológicas em pacientes com diabetes mellitus que foram infectados pelo SARS-CoV-2. A pesquisa bibliográfica foi realizada através de uma revisão da literatura utilizando os bancos de dados: Pubmed, Scielo e Google Acadêmico. Para efetuar a pesquisa, foram utilizados os seguintes descritores "Diabetes mellitus", "COVID-19", "periodontite" e "complicações odontológicas", e suas variantes em inglês. Como critério de inclusão foram utilizadas publicações dos últimos 3 anos, salvo artigos conceituais relevantes para a temática. Foram excluídos da análise textos que, após leitura do resumo, não abordavam o tema em estudo, apesar de conterem os descritores.

\section{RESULTADOS E DISCUSSÕES}

A doença causada pela infecção com SARS-CoV-2 (COVID-19), originou-se no Sul da China no final de 2019 e rapidamente se espalhou por diversos países em todo o mundo, causando a pandemia da COVID-19 (ZHU et al., 2020; WANG et al., 2020). A idade é um dos fatores de risco para o desenvolvimento de sintomas graves de 
COVID-19 (ZHOU et al., 2020), assim, pessoas com mais de 65 anos são especialmente vulneráveis à morbidade e mortalidade devido à infecção com SARSCoV-2. No entanto, pessoas com doença pulmonar crônica, obesidade grave, DM, doença renal crônica e doenças hepáticas também apresentam alto risco de desenvolver COVID-19 grave. Um estudo lista a hipertensão, obesidade e DM como as três principais condições subjacentes com resultados desfavoráveis para pacientes com COVD-19 que requerem hospitalização (RICHARDSON et al., 2020).

Embora a COVID-19 possa afetar diferentes órgãos do corpo, incluindo os rins e o fígado (LI et al., 2020), a principal causa de mortalidade é devido à capacidade do SARS-CoV-2 de infectar o trato respiratório, levando a pneumonia grave (REN et al., 2020).

Nos últimos anos, ficou evidente que a saúde bucal tem um grande impacto na saúde geral. Vários estudos sugerem que citocinas ou produtos microbianos liberados sistemicamente em resposta à infecção oral causam inflamações em órgãos distantes, o que aumenta o desenvolvimento de doenças sistêmicas, como a doença de Alzheimer, DM, doença cardíaca aterosclerótica e doença cerebrovascular (BUI et al., 2019; WU; NAKANISHI, 2014; JEPSEN et al., 2015; KHUMAEDI et al., 2015).

A periodontite grave é caracterizada pela destruição do periodonto, composto por tecidos, ligamentos e alguns ossos específicos (SLOTS, 2017). Esta doença é caracterizada por uma inflamação crônica não resolvida, em resposta a uma disbiose no biofilme subgengival (CURTIS; DIAZ; VANDYKE, 2020). A inflamação crônica leva frequentemente a inflamação sistêmica de baixo grau e aumento dos níveis de citocinas, como o fator de necrose tumoral alfa (TNF- $\alpha$ ), interleucina (IL)-1 $1 \beta$, IL-4, IL6 e IL-10 (ACHARYA et al., 2017), assim como a proteína C reativa (PCR) e ferritina (THOUNAOJAM, 2019).

Wu e Yang demonstraram que existe um aumento de células produtoras de IL17 no tecido gengival de pacientes que sofrem com periodontite, em comparação com pacientes saudáveis. Estes níveis aumentados foram encontrados também no soro de pacientes que sofrem com periodontite (WU; YANG, 2020).

Pacientes com COVID-19 grave e síndrome respiratória aguda grave (SARS) (MEHTA et al., 2020), geralmente apresentam uma exacerbada resposta imunológica, 
caracterizada pelo aumento nos níveis de citocinas pró-inflamatórias e dano generalizado nos tecidos, a chamada tempestade de citocinas (YANG et al., 2020a).

Pacientes com periodontite existente, antes da infecção por SARS-CoV-2, são propensos a terem níveis elevados de citocinas e podem ser suscetíveis a resultados graves e fatais. Os tecidos pulmonares de pacientes com COVID-19 expressam as citocinas pró-inflamatórias que desempenham papel essencial no desenvolvimento da periodontite ( YE; WANG; MAO, 2020).

Pitones-Rubio e colaboradores mostraram que a doença periodontal (DP) pode ser uma complicação causada pelo DM, através de um descontrole nos níveis de glicemia, e que o DM aumenta o risco de desenvolver DP. Além disso, os fatores externos e internos do hospedeiro influenciam a resposta imune na DP e COVID-19. Em pacientes hospitalizados com COVID-19 grave, foi confirmado que a intubação prejudicou a saúde bucal (PITONES-RUBIO et al., 2020).

A DP pode aumentar os níveis de glicose no sangue, levando a longos períodos de tempo em que o corpo está funcionando em condições hiperglicêmicas, o que prejudica a função do sistema imunológico inato e pode aumentar a replicação viral (PFÜTZNER; LAZZARA; JANTZ, 2020).

Um estudo de longo prazo, forneceu evidências para a importância da higiene bucal na prevenção primária da pneumonia em pessoas com DM. Os pesquisadores analisaram esta associação em 98.800 pessoas em Taiwan durante um período de 12 anos. Os autores concluíram que pacientes que receberam tratamento periodontal intensivo tiveram um risco médio de $66 \%$ menor de desenvolver pneumonia. Pacientes com DM tiveram um risco $78 \%$ maior de desenvolver pneumonia em comparação com o grupo controle (YANG et al., 2020b). Estas descobertas indicam que o paciente multi mórbido com DM e periodontite tem um risco notavelmente alto de pneumonia, mesmo sem infecção por SARS-CoV-2 (PFÜTZNER; LAZZARA; JANTZ, 2020).

Teeuw e colaboradores descreveram que existem evidências que o tratamento periodontal leva a uma melhora do controle glicêmico em pacientes com DM tipo 2 (TEEUW; GERDES; LOOS, 2010), e da síndrome metabólica (MONTERO et al., 2020). O tratamento da periodontite também melhora o metabolismo de lipídios e 
glicose (TEEUW et al., 2014), e os biomarcadores associados a aterosclerose, como a PCR sérica, IL-6, níveis de fibrinogênio e IL-1ß (D'AIUTO; ORLANDI; GUNSOLLEY, 2013).

Em um estudo realizado por Marouf e colaboradores, onde foram incluídos 568 pacientes, 258 apresentavam periodontite. Entre estes pacientes que apresentavam periodontite, 33 apresentaram complicações, enquanto apenas 7 dos 310 pacientes que não apresentavam periodontite apresentaram complicações pela COVID-19. A periodontite foi associada significativamente ao aumento do risco de complicações gerais em pacientes com COVID-19, bem como complicações na UTI (unidade de tratamento intensivo) em pacientes diabéticos e fumantes (MAROUF et al., 2021).

Um estudo realizado por Coke e colaboradores, descreve que os pacientes com DM têm maior probabilidade de apresentarem sintomas graves e complicações decorrentes da infecção por COVID-19, do que pacientes sem DM. Observaram também que a periodontite pode ser uma condição pré-existente que exacerba os resultados negativos da COVID-19. A tempestade de citocinas causada pela periodontite, tem sido associada a resultados adversos em infecções pelo COVID-19, como SARS e falência de múltiplos órgãos (COKE et al., 2021).

A DP presente em pacientes hospitalizados, idosos e em pacientes internados na UTI, leva a maior propensão a desenvolver a pneumonia adquirida em hospitais, o que leva a graves complicações relacionadas ao COVID-19 e ao DM (SAMPSON; KAMONA; SAMPSON, 2020).

Um ensaio clínico randomizado, realizado no Japão, investigou se a melhora da higiene oral reduziria a incidência de pneumonia e mortes relacionadas com pneumonia. Dos 417 pacientes que receberam higiene bucal após cada refeição, 11\% contraiu pneumonia, em comparação com 19\% do grupo que não recebeu higiene bucal. Além disso, a taxa de mortalidade por pneumonia no grupo controle foi quase o dobro do grupo prescrito com higiene bucal (SAMPSON; KAMONA; SAMPSON, 2020). A ligação entre bons hábitos de higiene bucal e um risco reduzido de infecções virais respiratórias agudas foram estabelecidas em outros estudos (ABE et al., 2006; IMSAND et al., 2002), incluindo uma revisão sistemática que concluiu que uma em 
cada dez mortes relacionadas à pneumonia em idosos poderiam ser evitadas melhorando a higiene oral (SJÖGREN et al., 2008).

A higiene oral deve ser mantida, se não melhorada, durante uma infecção por SARS-CoV-2, a fim de reduzir a carga bacteriana na boca e o risco potencial de uma superinfecção bacteriana. A higiene oral inadequada é considerada um fator de risco para complicações pós-virais, particularmente em pacientes já predispostos a biofilmes alterados, devido ao DM. Bactérias presentes em pacientes com COVID-19 grave estão associadas com a cavidade oral e a melhora da higiene oral pode desempenhar um papel na redução do risco de complicações (SAMPSON; KAMONA; SAMPSON, 2020).

Portanto, a correta higiene bucal irá reduzir a colonização orofaríngea e o risco de doenças respiratórias adquiridas, evitando complicações mais severas, o avanço e a propensão de desenvolver outras doenças sistêmicas como o DM e reduzindo a morbidade por COVID-19 (BOTROS; IYER; OJCIUS, 2020).

\section{CONCLUSÃO}

A maioria dos pacientes com complicações graves causadas pela COVID-19 tem condições subjacentes como obesidade, DM e hipertensão. Paralelamente, existem evidências crescentes de uma relação entre a periodontite e doenças sistêmicas não orais.

Observou-se que a cavidade oral também é um reservatório para patógenos respiratórios e pacientes com periodontite são mais propensos a desenvolver pneumonia adquirida em hospitais, quando comparado a indivíduos saudáveis. Foi possível constatar que a melhora na saúde bucal pode diminuir a gravidade dos sintomas de COVID-19 e reduzir a morbidade associada. Alguns estudos evidenciam que a periodontite associada ao COVID-19, em pacientes com DM, aumenta as possibilidades de complicações nestes pacientes.

Conclui-se que a compreensão desta associação ressalta a seriedade de manter a periodontite sob controle e a importância da higiene bucal meticulosa na pandemia da COVID-19. Também, os estudos apontam que o DM é um preditor 
EDUCAÇÃO, SAÚDE

ETECNOLOGIA

26 A 28 DE OUTUBRO DE 2021

\section{QUFN}

significativo de COVID-19 grave e doença periodontal, de modo que pode ser útil para identificar grupos de risco de COVID-19.

\section{AGRADECIMENTOS}

À Universidade Franciscana pelo apoio financeiro através da Bolsa de Projeto de Extensão - PROBEX/UFN. 


\section{QUFN}

\section{REFERÊNCIAS}

$A B E, S$. et al. Professional oral care reduced infuenza infection in elderly. Arch Gerontol Geriatr, v. 43, p. 157-164, 2006.

ACHARYA, A. B. et al. Cytokine ratios in chronic periodontitis and type 2 diabetes mellitus. Diabetes \& Metabolic Syndrome, v. 11, n. 4, p. 277-278, 2017.

BRANDÃO, D. F.; SILVA, A. G.; PENTEADO, L. A. M. Relação bidirecional entre a doença periodontal e a diabetes mellitus. Odontol. Clín.-Cient., v.10, n. 2, p. 117-120, 2011.

BUI, F. Q. et al. Association between periodontal pathogens and systemic disease. Biomedical Journal, v. 42, p. 27-35, 2019.

BOTROS, N.; IYER, p.; OJCIUS, D. M.Is there an association between oral health and severity of COVID-19 complications? Biomedical Journal, v. 43, p. 325-327, 2020.

COKE, C. J. et al. SARS-CoV-2 Infection and Oral Health: Therapeutic Opportunities and Challenges. J. Clin. Med., n. 10, p. 156, 2021.

CURTIS, M. A.; DIAZ, P. I.; VANDYKE, T. E. The role of the microbiota in periodontal disease. Periodontology, v. 83, p. 14-25, 2020.

D'AIUTO, F.; ORLANDI, M.; GUNSOLLEY, J. C. Evidence that periodontal treatment improves biomarkers and CVD outcomes. Journal of Clinical Periodontology, v. 40, n. 14, p. S85-105, 2013.

IMSAND, M. et al. Bronchopneumonia and oral health in hospitalised older patients. Gerodontology, v. 19, p. 66-72, 2002. 
JEPSEN, S. et al. Science transfer: oral health and general health - the links between periodontitis, atherosclerosis and diabetes. J Clin Periodontol, v. 42, p. 1071-1073, 2015.

KHUMAEDI, A.I. et al. The relationship of diabetes, periodontitis and cardiovascular disease. Diabetes Metab Syndr, v. 13, p. 1675-1678, 2019.

LI, M.Y. et al. Expression of the SARS-CoV-2 cell receptor gene ACE2 in a wide variety of human tissues. Infect Dis Poverty, v. 9, n. 1, p. 45, 2020.

MEHTA, P. et al. COVID-19: Consider cytokine storm syndromes and immunosuppression. The Lancet, v. 395, n. 10229, p. 1033- 1034, 2020.

MAROUF, N. et al. Association between periodontitis and severity of COVID-19 infection: A case-control study. Clinical Periodontology, v. 48, n. 4, p. 483-491, 2021.

MONTERO, E. et al. Impact of periodontal therapy on systemic markers of inflammation in patients with metabolic syndrome: A randomized clinical trial. Diabetes, Obesity \& Metabolism, v. 22, n. 11, p. 2120-2132, 2020.

PITONES-RUBIO, V. et al. Is periodontal disease a risk factor for severe COVID-19 illness?. Elsevier. Med Hypotheses, v. 144, p. 109969, 2020.

PFÜTZNER, A.; LAZZARA, M.; JANTZ, J. Why Do People With Diabetes Have a High Risk for Severe COVID-19 Disease?-A Dental Hypothesis and Possible Prevention Strategy. Journal of Diabetes Science and Technology, v. 14, n., 4, p. 769-771, 2020.

REN, L. L. et al. Identification of a novel coronavirus causing severe pneumonia in human: a descriptive study. Chin Med J, v. 133, n. 9, p. 1015-1024, 2020. 
RICHARDSON, S. et al. Presenting characteristics, comorbidities, and outcomes among 5700 patients hospitalized with COVID-19 in the New York city area. JAMA, v. 323, n. 20, p. 2052-2059, 2020.

SAMPSON, V.; KAMONA, N.; SAMPSON, A. Could there be a link between oral hygiene and the severity of SARS-CoV-2 infections? Br Dent J., v. 12, p. 971-975, 2020.

SJÖGREN, P. et al. A systematic review of the preventative effect of oral hygiene on pneumonia and respiratory tract infection in elderly people in hospitals and nursing homes: effect estimates and methodological quality of randomised controlled trials. $\mathbf{J}$ Am Geriatr Soc, v. 56, p. 2124-2130, 2008.

SLOTS, J. Periodontitis: Facts, fallacies and the future. Periodontology, v. 75, p. 723, 2017.

SOCIEDADE BRASILEIRA DE DIABETES. Diretrizes Sociedade Brasileira de Diabetes 2019-2020. São Paulo: Editora Clannad, 2019.

TEEUW, W. J. et al. Treatment of periodontitis improves the atherosclerotic profile: A systematic review and meta-analysis. Journal of Clinical Periodontology, v. 41, p. 70-79, 2014.

TEEUW, W. J.; GERDES, V. E. A.; LOOS, B. G. Effect of periodontal treatment on glycemic control of diabetic patients: A systematic review and meta-analysis. Diabetes Care, v. 33, n. 2, p. 421-427, 2010.

THOUNAOJAM, N. Effects of chronic periodontitis in serum ferritin levels before and 1 month after nonsurgical periodontal therapy: An intervention study. International Journal of Preventive and Clinical Dental Research, v. 6, n. 2, p. 32-34, 2019. 
WANG, C. et al. A novel coronavirus outbreak of global health concern. The Lancet, v. 395, n. 10223, p. $470-473,2020$.

WU, D.; YANG, X. O. TH17 responses in cytokine storm of COVID-19: An emerging target of JAK2 inhibitor Fedratinib. J Microbiol Immunol Infect, v. 53, n. 3, p. 368370, 2020.

WU, Z.; NAKANISHI, H. Connection between periodontitis and Alzheimer's disease: possible roles of microglia and leptomeningeal cells. J Pharmacol Sci, v. 126, p. 913, 2014.

YANG, Y. et al. Plasma IP-10 and MCP-3 levels are highly associated with disease severity and predict the progression of COVID-19. Journal of Allergy and Clinical Immunology, v. 146, p. 119-127, 2020a.

YANG, L. C. et al. The association of periodontal treatment and decreased pneumonia: a nationwide population-based cohort study. Int J Environ Res Public Health, v. 17, n. 1, p. 3356, 2020b.

YE, Q.; WANG, B.; MAO, J. The pathogenesis and treatment of the 'Cytokine Storm' in COVID-19. J. Infect., v. 80, p. 607-613, 2020.

ZHOU, F. et al. Clinical course and risk factors for mortality of adult inpatients with COVID- 19 in Wuhan, China: a retrospective cohort study. The Lancet, v. 395, n. 10229, p. 1054-1062, 2020.

ZHU, N. et al. A Novel Coronavirus from Patients with Pneumonia in China, 2019. N Engl J Med, v. 382, p. 727-733, 2020. 